\title{
Extra-Articular Proximal Tibia fractures: Proximal tibia plate versus intramedullary nailing
}

\author{
Zulfikar Patel $^{1, *}$, Bhavya B. Shah ${ }^{2}$, Hriday Acharya ${ }^{3}$ \\ ${ }^{\mathbf{1}}$ Assistant Professor, ${ }^{\mathbf{2}, \mathbf{3}}$ Resident, Dept. of Orthopaedics, Civil Hospital, Ahmedabad \\ *Corresponding Author: \\ Email: dr.zulfikarpatel@yahoo.co.in
}

\begin{abstract}
Introduction: Extra-articular promixal tibial fracture make up to around $10 \%^{(1)}$ of all shaft tibia fractures which are most commonly due to high velocity injury. Both conservative and operative management options are available, but conservative management has resulted in many complications. Intramedullary nailing and minial invasive plating are the preferred techniques of the available modalities of operative management. There is not enough evidence regarding the superiority of one of the two techniques. The purpose of this study is to compare these different approaches based on hospital stay, blood loss, union rates, $\mathrm{mal} / \mathrm{non}$-union, post-operative range of motion of knee, period of non-weight bearing, infection rates and implant removal rate.

Material and Methods: This is a randomized prospective study conducted on 50 osteoporotic patients with extra-articular proximal tibia fractures treated with minimally invasive proximal tibial plating vs intramedullary nailing at Department of Orthopedics, BJ medical college, Civil hospital Ahmedabad between June 2016 and May 2017.

Observation and Results: Patients with intramedullary nailing had lesser duration of stay, lower cost, less infection rate, less implamt removal rate but higher malunion and union rates compared to plating patients. Operative time, time to full weight bearing and range of motion was found to be similar in both.

Conclusion: Intramedullary nailing is preferred operative modality over plating because of decreased hospital stay, faster union, decreased operative time and decreased infective rate. However there was no significant difference in range of motion of knee, rate of malunion and nonunion
\end{abstract}

Keywords: Proximal tibia fracture, Intramedullary nailing, Plating.

\section{Introduction}

Proximal tibia fractures are very common. They are classified as primarily into extra articular and intraarticular fractures. The AO classification is shown below.

Extra-articular promixal tibial fracture make up to around $10 \%^{1}$ of all shaft tibia fractures which are most commonly due to high velocity injury. Both conservative and operative management options are available for these fractures but conservative management have resulted in complications like non-union, malunion, stiffness of joints or rotational instability. So operative management is preferred over conservative management in these patients. Various options are available for operative management which include Im nailing, external fixation, plating or a combination of these. In recent times, Intramedullary nailing and minial invasive plating are the preferred techniques of the available modalities of operative management. There is not enough evidence regarding the superiority of one of the two techniques. The purpose of this study is to compare these different approaches based on hospital stay, blood loss, union rates, mal/non-union, post-operative range of motion of knee ,period of non weight bearing, infection rates and implant removal rate.

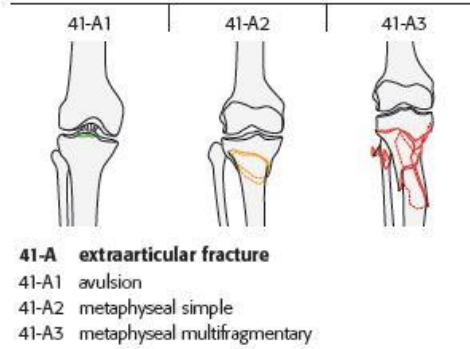

Fig. 1

\section{Material and Methods}

This is a randomized prospective study conducted on 50 osteoporotic patients with extra-articular proximal tibia fractures treated with minimally invasive proximal tibial plating vs intramedullary nailing at Department of
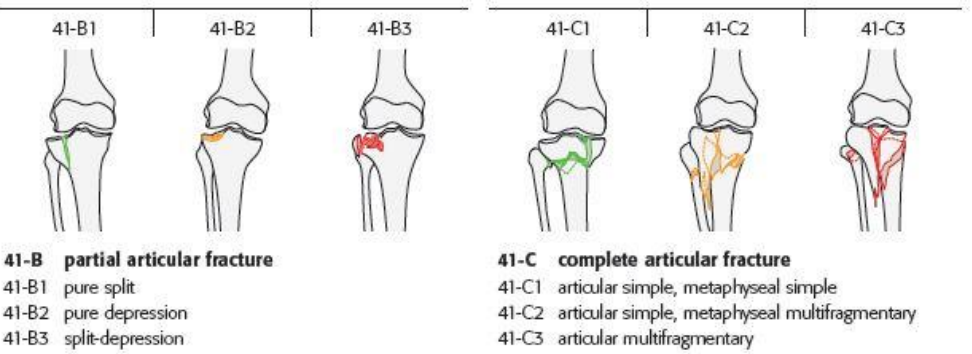

Orthopedics, BJ medical college, Civil hospital Ahmedabad between June 2016 and May 2017. Ethical committee approval was obtained. Consent was taken from the patients. 
Inclusion Criteria: In this study, patients having proximal tibial fracture with mature skeletal structure were included where proximal tibia is the region extending from the articular surface up to 1.5 times the medial to lateral width of the articular surface.

\section{Exclusion Criteria:}

1. Childrens and Skeletally immature patients

2. Intra articular Fractures.

3. Open fractures

4. Pathological fractures

5. Diaphyseal fractures

6. Those with associated injuries in same or opposite limb.

\section{Observation and Results}

a. Preoperative characteristics like age, sex, classification, mode of injury and time period from injury to operation were comparable in both groups.

b. Patients treated with intramedullary nailing has significant lesser time for postoperative hospital stay, full weight-bearing and union as compared to those managed with proximal plating.

c. Three patients with intramedullary nailing had delayed union for which dynamization was done at one month interval while two patients had nonunion for which exchange nailing + bone grafting was performed and in the other patient plating + bone grafting was done.

d. There was non-union in one patient in tibial plating for which bone grafting was performed.

e. All fractures united after revision surgery.

f. There was no significant difference in the alignment of fracture between the 2 groups.

g. Both the groups showed a varus inclination which was not significant (measuring an average of 3 degree in intramedullary nailing patients and 2.4 degree in those treated with plating).

h. The average range of motion was same in both the groups ranging from $85-150$ degree.

i. There was complain of pain in anterior aspect of knee in $10(20 \%)$ patients treated with IM nailing as compared to only $2(4 \%)$ treated with plating.

Table 1

\begin{tabular}{|l|c|c|}
\hline \multicolumn{1}{|c|}{ Criteria's } & $\begin{array}{c}\text { Intramedullary } \\
\text { Nailing }\end{array}$ & Plating \\
\hline Age & 38 & 34 \\
\hline Operative time (mins) & 80 & 100 \\
\hline Hospital stay (days) & 2 & 4 \\
\hline $\begin{array}{l}\text { Union time (weeks) or } \\
\text { time required before full } \\
\text { weight-bearing (weeks) }\end{array}$ & 17 & 20 \\
\hline Infection & 1 & 5 \\
\hline Coronal plane & 3 & 2.4 \\
\hline Sagittal plane & 3 & 2.6 \\
\hline Range of motion of knee & 115 & 120 \\
$(85-150)$ & $(85-150)$ \\
\hline Delayed union/non-union & 3 & 1 \\
\hline
\end{tabular}

\section{Discussion}

Data regarding the comparative study of management protocol and criteria for extra articular proximal tibia fracture is very limited. The primary goal of this study was to compare the results of tibial nailing and minimally invasive plating from various aspects.

In our study Patients treated with intramedullary nailing had shorter hospital stay because of it being a closed procedure operated with a smaller incision length there by requiring lesser monitoring.

Studies suggest an early weight bearing in those patients treated with intramedullary nailing as compared to those treated with plating. However it is still not clear when to start weight bearing in these patients since it depends on a lot of factors ranging from fracture type, location, time of operation and most importantly surgeons preference.

In various studies full weight bearing is started between $0-16$ weeks in most of the patients treated in both the groups depending on fracture type, location, time of operation and surgeon's preference.

In our study full weight bearing was started after complete radiological union of the fracture was achieved. It was significantly lower in those treated with nailing (16 weeks) as compared to plating which was 20 weeks.

We started physiotherapy (SLR, SQE, Hip Abducation) in both the patients from day 2 so the range of movement in both the patients was found to be the same.

The infection rate in various studies was found to range from $0-10 \%$ in nailing and $0-15 \%^{2}$ in plating. But, in the study by Lindvall et al, the authors reported significantly higher infection rates: $28 \%$ in the nailing group and $24 \%$ in the plating group. The most probable reason for this is the higher proportion $(42.8 \%)$ of patients with open fractures in their study. In our study the infection rate was $2 \%$ in those treated with nailing and $10 \%$ in plating.

In patients who developed infection in post operative period were either treated with higher IV antibiotics or debridement. In 2 patients who had plating done had to be taken for debridement and removal of implant of which one patient had external fixator done after removal of plate.

The union rate in patients treated with both nailing and plating was found to be similar being $95 \%$ in the former and $97 \%$ in plating. Studies show a range of $90-$ $100 \%{ }^{3}$ which in lesser than our study because our study dint include open fractures.

Malunion is a common complication of the nailing in proximal tibia fractures and has been reported to occur in 3-100\% of cases in various studies. In our study, there was a rotational malalignmentin 4 patients (those having more than $5^{\circ}$ degree of rotational disalignment) in the nailing group, malalignment in varus in 1 case and anterior apex deformity in 4 patients. Various methods have been used to prevent malreduction like the use of 
polar screws, polar st pins, unicortical plating, a universal distractor, nailing in the semi-extended position, or the use of a nail with a more proximal Herzog bend (proximalbend). In our study, we used polar st pins in 2 patients and reduction plating in 1 patient. In a few cases, we used a reduction clamp to prevent proximal fragment extension while inserting the nail. Taking a higher entry in tibia while inserting the nail is a common technique used in proximal tibia fractures. This technique brought our insertion point more in line with the medullary canal of the tibia, hence reducing the extension of the proximal fragment. The plating group also had 5 patients of malunion (16\%), but the difference was not statistically significant.

An important character of proximal tibia fractures is gross swelling around the fracture site. It is one of the character which was encountered in $90 \%$ of the proximal tibia fractures presenting to us following fresh trauma. It was managed initially by distraction fixator at the site to reduced the swelling and the patients were called up in follow up after 2 weeks for definitive management. Patients were explained to apply a hygroscopic solution (gylcerine and magnesium sulphate) at the site and to keep the limb elevated at discharge. The is one of the most common initially management in most of the patients undergoing proximal tibia plating at our setup , while most of the patients planned for tibia nailing were operated immediately. These is one of those consideration which have not been mention in most of the literatures.

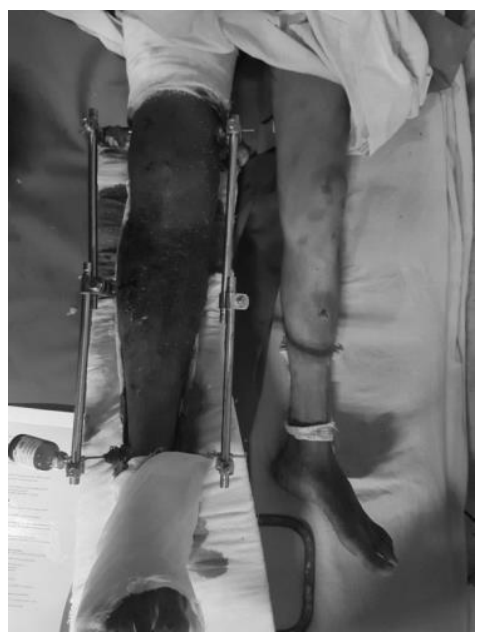

Fig. 2

The intramedullary nailing technique had an advantage in terms of lesser operative time, hospital stay, early weight bearing and early fracture union.
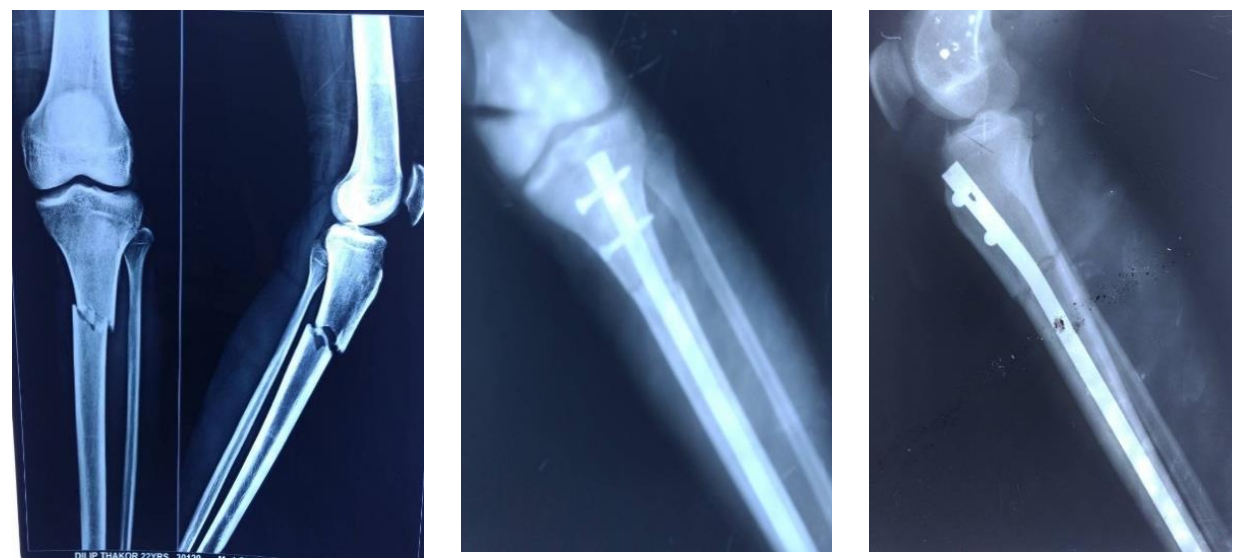

Fig. 3: Intramedullary Nailing 

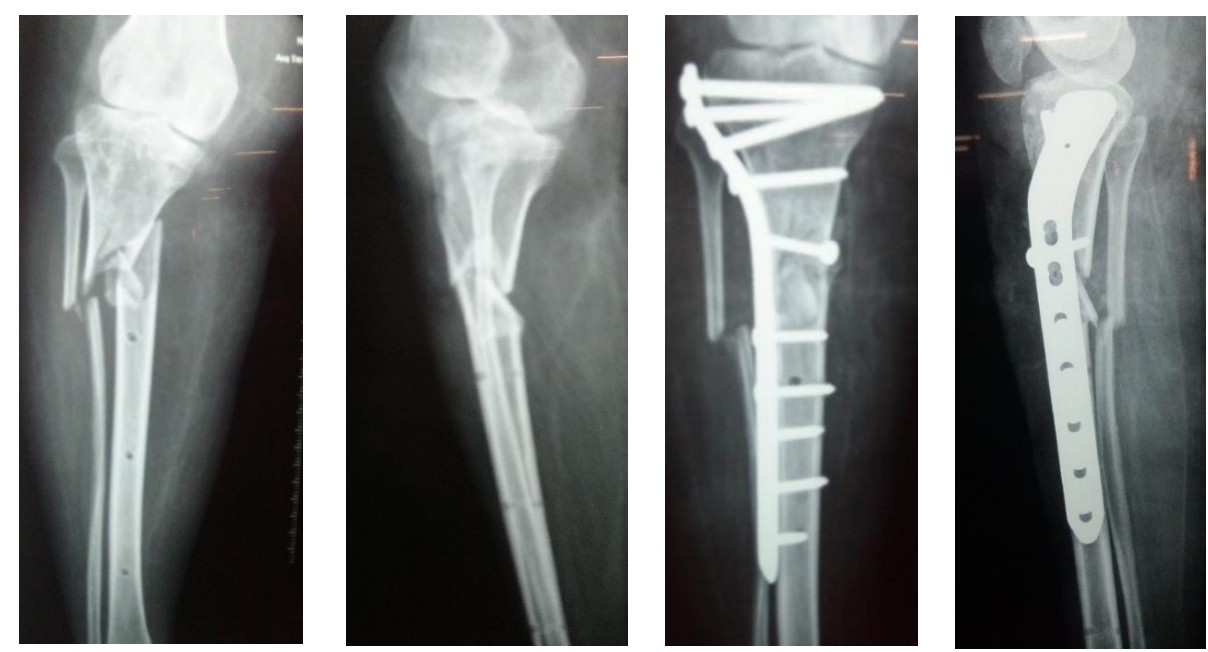

Fig. 4: Proximal Tibia Plating

\section{Conclusion}

Intramedullary nailing is preferred operative modality over plating because of decreased hospital stay, faster union, decreased operative time and decreased infective rate.

However there was no significant difference in range of motion of knee, rate of malunion and non-union

\section{Limitations of this study}

1. Small number of patients.

2. Inter surgeon variation.

3. Absence of long-term follow-up to evaluate the outcome of mal-alignment in terms of the development of osteoarthritis of the knee.

4. Use of different company of plates and different alloy used in plating. These had a difference in their association with the fracture site and local site reaction.

\section{References}

1. Court-Brown CM, McBirnie J. The epidemiology of tibial fractures. J Bone Jt Surg Br. 1995;77(3):417-421.

2. Bhandari M, Audige L, Ellis T. Operative treatment of extraarticular proximal tibial fractures. J Orthop Trauma. 2003;17(8):591-595. doi: 10.1097/00005131200309000-00013.

3. Lindvall E, Sanders R, Dipasquale T, Herscovici D, Haidukewych G, Sagi C. Intramedullary nailing versus percutaneous locked plating of extra-articular proximal tibial fractures: comparison of 56 cases. J Orthop Trauma. 2009;23:485-492. doi: 10.1097/BOT.0b013e3181b013d2

4. https://www2.aofoundation.org/wps/portal/surgery?show Page $=$ diagnosis $\&$ bone $=$ Tibia $\&$ segment $=$ Proximal . 